\title{
Fact Sheet: Telehealth
}

Advancing Health in America

\section{The Issue}

Telehealth connects patients to vital health care services through videoconferencing, remote monitoring, electronic consults and wireless communications. By increasing access to physicians and specialists, telehealth helps ensure patients receive the right care, at the right place, at the right time.

Currently, 76 percent of U.S. hospitals connect with patients and consulting practitioners at a distance through the use of video and other technology. Almost every state Medicaid program has some form of coverage for telehealth services, and private payers are embracing coverage for many telehealth services. However, there are barriers to wide adoption of telehealth. Medicare generally still limits coverage and payment for many telehealth services, lagging behind other payers. The Medicare program recently expanded coverage for telehealth services for stroke patients and substance use treatment in response to statutory changes. Medicare also expanded payments to clinicians for virtual check-ins. While promising, these incremental steps are not sufficient. In addition, limited access to adequate broadband services hampers the ability of some rural facilities to deploy telehealth. The challenge of cross-state licensure also looms as a major issue. Other policy and operational issues include credentialing and privileging, online prescribing, privacy and security and fraud and abuse. The federal government needs to do more to increase the use of telehealth.

\section{AHA Position}

The AHA supports the expansion of patient access created by hospitals' efforts to deliver high-quality and innovative telehealth services. Specifically, we are advocating for:

- Expansion of Medicare coverage with adequate reimbursement that takes into consideration the nursing and other costs incurred at the site where the patient is located (originating site). The Centers for Medicare \& Medicaid Services (CMS) also should include telehealth waivers in all of its demonstrations and adopt a more flexible approach to adding new telehealth services to Medicare.

- Resolution of legal and regulatory challenges that hinder the provision of telehealth services.

- Additional federal research on the cost-benefits of telehealth.

- Improved access to broadband technology for rural areas by improving the Federal Communications Commission (FCC) Rural Health Care Program. 
- Limited Medicare coverage impedes the expansion of telehealth services. Current statute restricts most telehealth services to patients located in rural areas and in specific settings (such as a hospital or physician office), covers only a limited number of services, and allows only real-time, two-way video conference capabilities, with limited exceptions, such as telestroke. Changes needed include: widespread elimination of geographic and setting locations requirements so patients outside of rural areas can benefit from telehealth; expanding the types of technology that can be used, including remote monitoring; and covering all services that are safe to provide, rather than a small list of approved services.

- Significant federal and state legal and regulatory issues will determine whether and how providers can offer specific telehealth services. In general, the provision of telehealth services requires compliance with an array of federal and state rules. Legal and regulatory challenges abound in the following areas:

- Coverage and payment;

- Health professional licensure;

- Credentialing and privileging;

- Online prescribing, including for controlled substances;

- Medical malpractice and professional liability insurance;

- Privacy and security; and

- Fraud and abuse.

- Additional research is needed to evaluate telehealth. Research and experience under the Medicare program suggest that policymakers' concerns about increased access to telehealth leading to increased spending may be overstated, particularly when weighed against the potential benefits in quality, patient experience and efficiency. However, there are insufficient studies on the cost-benefits of telehealth outside of a limited number of services, such as telestroke. More and better research is needed for other conditions and newer technologies, such as remote monitoring of patients.

- Value-based care requires telehealth. The health care field is quickly moving from fee-for-service to a value-based delivery system. Success in new payment models, such as bundling, accountable care organizations (ACOs) and new physician payment models, requires flexibility to deploy telehealth, particularly as part of care management programs. CMS has shown some willingness to provide waivers, and Congress has expanded the ability of some ACOs to use telehealth, but only in limited circumstances.

- Federal programs to expand broadband need to be simplified. According to the FCC, 34 million Americans still lack access to adequate broadband. And, there is a large digital divide, with almost 40 percent of those living in rural areas lacking access. The FCC's Rural Health Care Program supports broadband adoption, but it is administratively burdensome and provides an insufficient level of subsidy for remote health care providers. While the FCC has taken positive steps by increasing the subsidy cap, and proposing a Connected Care Pilot, we need even greater federal investment in broadband access, particularly in rural areas. 
- State policymakers are increasingly focusing their attention on telehealth. Today, 35 states and the District of Columbia have enacted "parity" laws, which generally require health insurers to cover services provided via telehealth the same way they would reimburse for services provided in person. States vary on payment parity. We believe all payers should provide payment parity with services delivered in person.

- Medicare reimbursement for telehealth services does not provide adequate compensation to all sites of care. The professional providing the service, who is at a distant site, is paid under the Medicare fee schedule; however, the facility where the patient is located (originating site) is paid a small "originating" fee of about $\$ 25$ to $\$ 30$. While the Medicare statute does not specify which facilities may serve as distant site, CMS has excluded rural health clinics and federally qualified health centers.

- Telehealth helps increase health care value and affordability. Virtual care technology saves patients time and money, reduces patient transfers, emergency department and urgent care center visits, and delivers savings to payers. In addition, telehealth helps address physician burnout by reducing clinicians' drive times and allowing more time for patients.

- Hospitals are increasingly using telehealth (see charts above).

For more resources on telehealth, including case studies and research reports, visit www.aha.org/telehealth. 